\title{
HEART RATE DYNAMICS DURING A TREADMILL CARDIOPULMONARY EXERCISE TEST IN OPTIMIZED BETA-BLOCKED HEART FAILURE PATIENTS
}

\author{
Vitor Oliveira Carvalho, Guilherme Veiga Guimarães, Emmanuel Gomes \\ Ciolac, Edimar Alcides Bocchi
}

doi: $10.1590 / \mathrm{S1807-59322008000400011}$

Carvalho VO, Guimarães GV, Ciolac EG, Bocchi EA. Heart rate dynamics during a treadmill cardiopulmonary exercise test in optimized beta-blocked heart failure patients. Clinics. 2008;63:479-82.

BACKGROUND: Calculating the maximum heart rate for age is one method to characterize the maximum effort of an individual. Although this method is commonly used, little is known about heart rate dynamics in optimized beta-blocked heart failure patients. AIM: The aim of this study was to evaluate heart rate dynamics (basal, peak and \% heart rate increase) in optimized beta-blocked heart failure patients compared to sedentary, normal individuals (controls) during a treadmill cardiopulmonary exercise test.

METHODS: Twenty-five heart failure patients ( $49 \pm 11$ years, $76 \%$ male), with an average LVEF of $30 \pm 7 \%$, and fourteen controls were included in the study. Patients with atrial fibrillation, a pacemaker or noncardiovascular functional limitations or whose drug therapy was not optimized were excluded. Optimization was considered to be $50 \mathrm{mg} /$ day or more of carvedilol, with a basal heart rate between 50 to $60 \mathrm{bpm}$ that was maintained for 3 months.

RESULTS: Basal heart rate was lower in heart failure patients $(57 \pm 3 \mathrm{bpm})$ compared to controls $(89 \pm 14 \mathrm{bpm}$; $\mathrm{p}<0.0001)$. Similarly, the peak heart rate (\% maximum predicted for age) was lower in HF patients $(65.4 \pm 11.1 \%)$ compared to controls $(98.6 \pm 2.2$; $\mathrm{p}<0.0001)$. Maximum respiratory exchange ratio did not differ between the groups $(1.2 \pm 0.5$ for controls and $1.15 \pm 1$ for heart failure patients; $\mathrm{p}=0.42$ ). All controls reached the maximum heart rate for their age, while no patients in the heart failure group reached the maximum. Moreover, the $\%$ increase of heart rate from rest to peak exercise between heart failure $(48 \pm 9 \%)$ and control $(53 \pm 8 \%)$ was not different $(\mathrm{p}=0.157)$.

CONCLUSION: No patient in the heart failure group reached the maximum heart rate for their age during a treadmill cardiopulmonary exercise test, despite the fact that the percentage increase of heart rate was similar to sedentary normal subjects. A heart rate increase in optimized beta-blocked heart failure patients during cardiopulmonary exercise test over $65 \%$ of the maximum age-adjusted value should be considered an effort near the maximum. This information may be useful in rehabilitation programs and ischemic tests, although further studies are required.

KEYWORDS: Heart failure. Heart rate. Carvedilol. Exercise. Optimization.

\section{BACKGROUND}

Heart failure (HF) is considered the last stage of heart disease and a significant cause of worldwide mortality and morbidity. ${ }^{1}$ In recent years, beta-blocking therapy has become a primary pharmacologic intervention in patients

Laboratório de Insuficiência Cardíaca e Transplante do Instituto do Coração, Faculdade de Medicina da Universidade de São Paulo (INCOR - HCFMUSP) - São Paulo/SP, Brazil.

Email: vitor.carvalho@usp.br

Received for publication on May 02, 2008

Accepted for publication on May 07, 2008 with HF. The prescription of beta-blockers arises from an abundance of evidence demonstrating that beta-blocking therapy improves survival and reduces hospitalization ${ }^{2}$, although improvement in aerobic capacity has not been proved. $^{3}$

Cardiopulmonary exercise testing is a well-established technique to evaluate peak oxygen consumption $\left(\mathrm{pVO}_{2}\right)$ in HF patients. This variable is the best prognostic factor in HF patients, even in the beta-blocking era. ${ }^{4}$ Aerobic exercise training is also a well-established nonpharmacological method to increase $\mathrm{pVO}_{2}$ in $\mathrm{HF}$ patients. In the HF population, prescription of adequate aerobic effort relative 
Table 1 - Subject characteristics

\begin{tabular}{lccc}
\hline & Heart Failure (25) & Controls (14) & $\mathrm{p}$-value \\
\hline Age & $49 \pm 11$ years & $30 \pm 6$ years & $<0.0001$ \\
Peak oxygen consumption & $19 \pm 6 \mathrm{mLO}_{2} \cdot \mathrm{kg}^{-1} \cdot \mathrm{min}^{-1}$ & $31 \pm 4 \mathrm{~mL} \mathrm{O}_{2} \mathrm{~kg}^{-1} \mathrm{mim}^{-1}$ & $<0.0001$ \\
Body mass index & $23 \pm 2 \mathrm{~kg} / \mathrm{m}^{2}$ & $22 \pm 1 \mathrm{~kg} / \mathrm{m}^{2}$ & $\mathrm{p}=0.18$ \\
Basal HR & $57 \pm 3 \mathrm{bpm}$ & $89 \pm 14 \mathrm{bpm}$ & $<0.0001$ \\
Peak HR (\% maximum predicted for age) & $65.4 \pm 11.1 \%$ & $98.6 \pm 2.2 \%$ & $<0.0001$ \\
HR percentage increase & $48 \pm 9 \%$ & $53 \pm 8 \%$ & $\mathrm{p}=0.157$ \\
\hline
\end{tabular}

to intensity based on heart rate (HR) is crucial to obtain both an increase in exercise capacity and a reasonable control of exercise-related risks. ${ }^{1}$

The maximum HR adjusted for age $(220$ - age $)$ is commonly used to characterize a maximum effort in normal subjects. ${ }^{5}$ Although the HR dynamics are known in non-optimized carvedilol HF patients ${ }^{6}$, little is known in optimized beta-blocked HF patients. The aim of this study is to evaluate HR dynamics (basal, peak and the percentage increase of HR) in optimized beta-blocked HF patients compared to normal individuals during a treadmill cardiopulmonary exercise test.

\section{MATERIALS AND METHODS}

\section{Study population}

A total of 25 ( $76 \%$ male, $24 \%$ female) HF patients (49 \pm 11 years) having an average left ventricle ejection fraction of $30 \pm 7 \%$ (determined by echocardiography), and 14 (36\% male, 64\% female) sedentary normal individuals (control group) (30 \pm 6 years) were included in the study. Data were collected from March 2006 to September 2007. HF patient characteristics are listed in Tables 1 and 2. Patients with atrial fibrillation, a pacemaker, or noncardiovascular functional limitations such as osteoarthritis, chronic obstructive pulmonary disease and patients whose drug therapy was not optimized were excluded from this study. Optimization was considered to be $50 \mathrm{mg} /$ day or more of carvedilol and a resting HR between 50 and 60 beats per minute, maintained for at least 3 months. ${ }^{7}$

This protocol was approved by the Ethical Committee of Instituto do Coração do Hospital das Clínicas da Faculdade de Medicina da Universidade de São Paulo (InCor HCFMUSP). All patients provided informed consent prior to participation.

\section{Cardiopulmonary exercise test}

Patients and normal subjects were asked to refrain from both strenuous physical activities and the consumption of any stimulants (coffee, tobacco, alcohol) that could influence
Table 2 - Characteristics of heart failure patients

\begin{tabular}{ll}
\hline Characteristics of heart failure patients & $\begin{array}{l}\text { Number of patients } \\
(\%), \text { dose }(\mathrm{mg} / \text { day })\end{array}$ \\
\hline Etiology: & $42 \%$ \\
$\quad$ Ischemic & $44 \%$ \\
$\quad$ Idiopathic dilated cardiomyopathy & $14 \%$ \\
$\quad$ Hypertensive & \\
NYHA functional class: & $42 \%$ \\
$\quad$ I & $42 \%$ \\
$\quad$ II & $16 \%$ \\
$\quad$ III & $30 \pm 7 \%$ \\
Left ventricle ejection fraction & \\
Current medications: & \\
Diuretics & $64 \%, 52 \pm 30 \mathrm{mg} /$ day \\
$\quad$ Furosemide & $48 \%, 43 \pm 24 \mathrm{md} /$ day \\
$\quad$ hydrochlorothiazide & \\
ACE inhibitors & $64 \%, 38 \pm 6 \mathrm{mg} /$ day \\
$\quad$ Enalapril & $20 \%, 93 \pm 37 \mathrm{mg} /$ day \\
$\quad$ Captopril & $16 \%, 75 \pm 29 \mathrm{mg} /$ day \\
Angiotensin II AT1 receptor antagonists & \\
(losartan) & $100 \%, 50 \pm 0 \mathrm{mg} /$ day \\
$\beta$-adrenergic receptor blocker (carvedilol) & $28 \%, 25 \pm 0 \mathrm{mg} /$ day \\
Spironolactone & $44 \%, 0.25 \pm 0 \mathrm{mg} / \mathrm{day}$ \\
Digoxin & $20 \%, 56 \pm 35 \mathrm{mg} /$ day \\
\hline Isosorbide 5-mononitrate &
\end{tabular}

heart rate for 24 hours prior to the cardiopulmonary exercise test. The patients' last meals were ingested at least 2 hours before the start of the test. All subjects underwent the cardiopulmonary exercise test on a programmable treadmill (Series 2000; Marquette Electronics; Milwaukee, WI, USA) in a temperature-controlled room $\left(21-23^{\circ} \mathrm{C}\right)$ between 10:00 a.m. and 15:00 p.m. with a standard 12 lead continuous electrocardiogram monitor (Max 1; Marquette Electronics). Blood pressure monitoring was performed by the auscultation method. Minute ventilation (VE), oxygen uptake $\left(\mathrm{VO}_{2}\right)$, carbon dioxide output $\left(\mathrm{VCO}_{2}\right)$ and other cardiopulmonary variables were acquired breath-by-breath by a computerized system (Vmax 229 model, SensorMedics, Yorba Linda, CA, USA). Resting $\mathrm{VO}_{2}$ and $\mathrm{HR}$ were computed as the mean of the final 30 seconds of the resting period, while peak effort $\mathrm{pVO}_{2}$ and heart rate (peakHR) were the mean values of the final 30 seconds of effort before exhaustion. The respiratory 
exchange ratios (RER) were recorded as the averaged samples obtained during each stage of the protocol in both HF patients and normal subjects (Naughton and Balke 4.0, respectively). A satisfactory cardiopulmonary exercise test was characterized by RER $>1.05$ and symptoms of maximum effort. Maximum HR predicted for age was calculated by the equation: 220 - age.

\section{Current medication intake}

All HF patients were receiving a beta-blocker (carvedilol) associated with ACE inhibitors (enalapril) or angiotensin II AT1 receptor antagonists (losartan). The medication profile of the HF patient group is shown in Table 2. Patients took beta-blockers, ACE inhibitors, Angiotensin II AT1 receptor antagonists and Isosorbide 5-mononitrate two times per day, one half of the daily dose in the morning (9:00 a.m.) and the other half at night (9:00 p.m.). Diuretics, digoxin and spironolactone were taken in the morning (9:00 a.m.).

\section{STATISTICAL ANALYSIS}

The descriptive analysis was presented as the mean, standard deviation and 95\% Confidence Interval (CI). The basal HR, percentage HR increase during cardiopulmonary test and peak HR (\% maximum predicted for age) for heart failure patients and controls were normally distributed. To compare these variables, we used the unpaired student's $t$-test.

Data were analyzed using the Statistical Package for Social Sciences (SPSS) for Windows, v 11.5 (SPSS Inc, Chicago, Ill). Statistical significance was set at $\mathrm{p}<0.05$.

\section{RESULTS}

All subjects performed a satisfactory cardiopulmonary exercise test (RER $1.2 \pm 0.5$ in normals and $1.15 \pm 1$ in HF patients; $\mathrm{p}=0.42$ ). The basal HR was lower in optimized beta-blocked HF patients $(57 \pm 3 \mathrm{bpm})$ compared to controls $(89 \pm 14 \mathrm{bpm})(\mathrm{p}<0.0001)$, as also seen with peak HR $(\%$ maximum predicted for age) $(65.4 \pm 11.1 \%$ for $\mathrm{HF}$ and $98.6 \pm 2.2$ for controls; $p<0.0001)$. All controls reached the maximum HR for their age, while no patients in the HF group did. Moreover, the percentage increase of HR from rest to peak exercise between HF $(48 \pm 9 \%)$ and control groups $(53 \pm 8 \%)$ was not different $(\mathrm{p}=0.157)$ (Figure 1).

\section{DISCUSSION}

The main finding of this study is the observation that all controls reached the maximum HR for their age, while none of the patients in the HF group were able to do so. Moreover, no difference was seen between the percentage increase of HR from rest to peak exercise between HF and control groups.

HR variability has been studied as a non-invasive tool to assess cardiac autonomic control of the nervous system, and it is proposed to reflect the interaction of sympathetic and parasympathetic (vagal) activity. ${ }^{8}$ Exercise training in

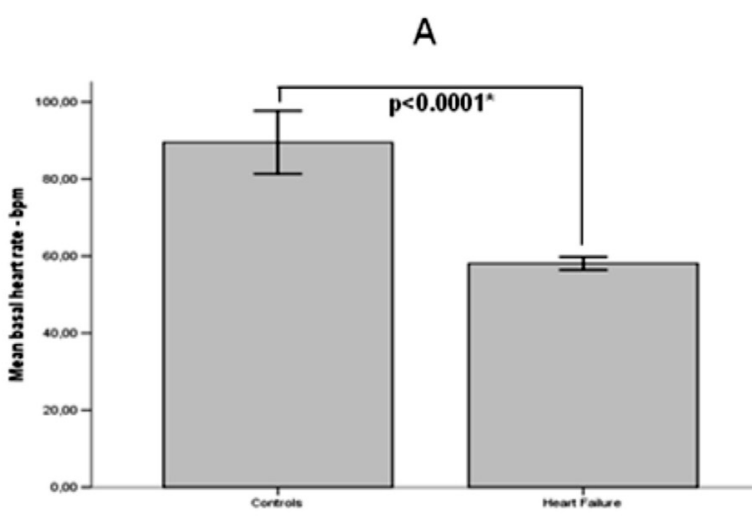

B

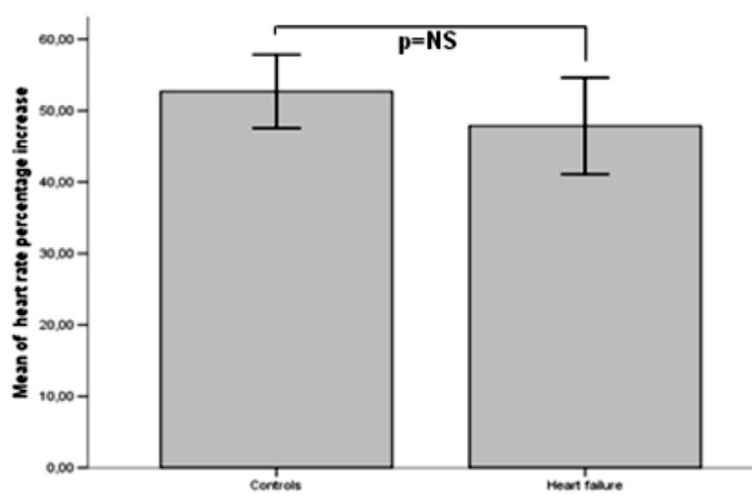

C

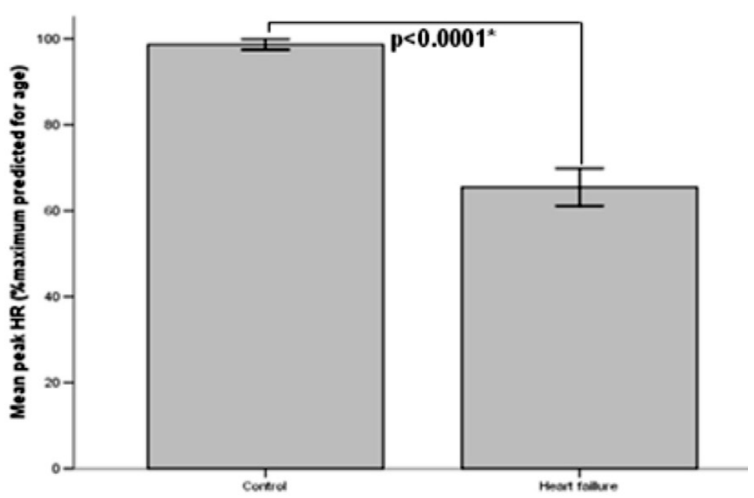

Mean $\pm 95 \% \mathrm{Cl}$

Figure I - Data are presented by mean $\pm 95 \%$ of conndence interval. A: Basal HR between controls and heart failure group; B: Mean of HR percentage increase between controls and heart failure group and $\mathrm{C}$ : Mean of peak HR (\%maximum predicted for age). * significant values $(<0.05)$ 
sedentary subjects affects this control, as carvedilol do in HF patients. ${ }^{9}$ The HR dynamics in optimized HF patients and the percentage of HR increase during the cardiopulmonary exercise test is unknown. Achievement of age-predicted values for maximal HR during exercise is often used as a reflection of maximal or near maximal effort $\left(220\right.$ - age). ${ }^{5}$ This method has been questioned by some authors, ${ }^{10}$ but it is currently the most commonly-used method in the world.

In our study, all sedentary subjects in the normal group reached the maximum HR for the age in the cardiopulmonary exercise test, while no patients in the HF group were able to reach. HR increased by only $65 \%$ of the maximum agepredicted HR in the HF group and $98 \%$ in the normal group. This is in partial agreement with Mezzani et al, who observed a HR increase of $76 \%$ of the maximum age-predicted in their non-optimized beta-blocker dose HF group and a $93 \%$ of the maximum age-predicted increase in the control group. The difference in HR increase in HF patients observed by Mezzani et al and our study is attributed to carvedilol dose optimization. The HR increase in the normal group of his study was similar to our results, as expected. Brawner et al designed a study to test the hypothesis that the percentage of HR reserve (peak HR - rest HR) was equivalent to the percentage of $\mathrm{VO}_{2}$ reserve during treadmill exercise among patients with a history of myocardial infarction, a group of patients with $\mathrm{HF}$ and a group with subjects with risk factors for heart diseases. In this study, the authors showed that the peak HR was higher in non-beta-blocked HF patients than in beta-blocked patients $(143 \pm 3 \mathrm{bpm}$ versus $125 \pm 4 \mathrm{bpm}$ $\mathrm{p}<0.01)$, while the HR reserve was the same $(52 \pm 3$ versus $50 \pm 3, p=N S)$. The authors did not calculate the percentage of HR increase or compare any of these variables with the cardiovascular risk factors group. ${ }^{11}$

Our data suggest that the maximum HR predicted for age (220 - age) should not be used to characterize a maximum effort in optimized beta-blocked HF patients. Despite this, the percentage increase of HR from rest to peak exercise between HF and control groups was not different. Thus, a peak HR of $65 \%$ of the maximum HR predicted for age should be considered to characterize and estimate a nearmaximal effort in HF patients undergoing optimized betablocking therapy, rather than the currently used definition of 90 to $100 \%$ of 220 -age. This equation (65\% (220 - age)) has never been evaluated previously. We hypothesized that the HR percentage increase was the same in HF patients and controls because the optimized beta-blocked HF patients started the cardiopulmonary exercise test with a basal HR (effect of carvedilol) lower than the normal group, despite the fact that the maximum HR calculated by age was not reached.

\section{CONCLUSION}

In the treadmill cardiopulmonary exercise test, optimized beta-blocked HF patients had both basal and peak HRs that were lower than control. No patient in the HF group reached the maximum HR predicted by the age, despite the fact that the percentage HR increase was the same as in sedentary normal subjects. In HF patients undergoing optimized beta-blocking therapy, the maximum HR during cardiopulmonary exercise test should be set as $65 \%$ of 220 - age to characterize and estimate near-maximal effort during the exercise treadmill test. This information may also be useful in rehabilitation programs and ischemic tests, although further studies are required.

\section{REFERENCES}

1. Working Group on Cardiac Rehabilitation \& Exercise Physiology and Working Group on Heart Failure of the European Society of Cardiology. Recommendations for exercise testing in chronic heart failure patients. Eur Heart J. 2001;22:37-45.

2. Lechat P, Packer M, Chalon S, Cucherat M, Arab T, Boissel JP. Clinical effects of beta-adrenergic blockade in chronic heart failure: a meta-analysis of double-blind, placebo-controlled, randomized trials. Circulation. 1998;98:1184-91.

3. Witte KK, Thackray S, Nikitin NP, Cleland JG, Clark AL. The effects of long-term beta-blockade on the ventilatory responses to exercise in chronic heart failure. Eur. J. Heart Fail. 2005;7:612-617.

4. O'Neill JO, Young JB, Pothier CE, Lauer MS. Peak oxygen consumption as a predictor of death in patients with heart failure receiving betablockers. Circulation. 2005;111:2313-18.

5. Task Force of the Italian Working Group on Cardiac Rehabilitation Prevention. Statement on cardiopulmonary exercise testing in chronic heart failure due to left ventricular dysfunction: recommendations for performance and interpretation Part I: Definition of cardiopulmonary exercise testing parameters for appropriate use in chronic heart failure. Eur J Cardiovasc Prev Rehabil. 2006;13:150-64.

6. Agarwal AK, Venugopalan P. Beneficial effect of carvedilol on heart rate response to exercise in digitalised patients with heart failure in atrial fibrillation due to idiopathic dilated cardiomyopathy. Eur J Heart Fail. 2001;3:437-40.

7. Packer M, Coats A, Fowler M, Katus H, Krum H, Mohacsi P et al. Effect of Carvedilol on Survival in Severe Chronic Heart Failure. 2001;334:1651-58.

8. Perini R, Veicsteinas A. Heart rate variability and autonomic activity at rest and during exercise in various physiological conditions. Eur J Appl Physiol. 2003;90:317-25.

9. Kasama S, Toyama T, Hatori T, Sumino H, Kumakura H, Takayama Y, et al. Evaluation of cardiac sympathetic nerve activity and left ventricular remodelling in patients with dilated cardiomyopathy on the treatment containing carvedilol. Eur Heart J. 2007;28:989-95.

10. Karavirta L, Tulppo MP, Nyman K, Laaksonen DE, Pullinen T, Laukkanen RT, et al. Estimation of maximal heart rate using the relationship between heart rate variability and exercise intensity in 40-67 years old men. Eur J Appl Physiol. 2008 Jan 11 [Epub ahead of print].

11. Clinton A Brawner, Steven J Keteyian, And Jonathan K Ehrman. The relationship of heart rate reserve to VO[sub2] reserve in patients with heart disease. Medicine and Science in Sports and Exercise. 2002;34:418-22. 